\title{
Search for Higgs bosons beyond the standard model in b-quark final states at the CMS experiment
}

\author{
Rainer Mankel* \\ Deutsches Elektronen-Synchrotron (DESY), Notkestr 85, D-22607 Hamburg, Germany \\ E-mail: Rainer. Mankeledesy.de \\ On behalf of the CMS collaboration
}

\begin{abstract}
While the existence of a Higgs boson with a mass near $125 \mathrm{GeV}$ has been clearly established, the detailed structure of the entire Higgs sector is yet unclear. Besides the standard model interpretation, well-motivated models with extended Higgs sectors are being considered. Such options include the minimal and next-to-minimal supersymmetric extensions (MSSM and NMSSM) of the standard model. Direct searches for additional Higgs bosons in b-quark final states are a promising way to address this key question. This article presents recent results from the CMS experiment.
\end{abstract}

The European Physical Society Conference on High Energy Physics 22-29 July 2015

Vienna, Austria

${ }^{*}$ Speaker. 


\section{Introduction}

The observation of a Higgs boson at a mass of about $125 \mathrm{GeV}$ by the ATLAS and CMS experiments $[1,2,3]$ opened a new era in the history of particle physics. The properties of this new state have been thoroughly investigated, and at the current level of precision found to be compatible with the predictions of the standard model (SM). Nevertheless, the observed boson could equally be only the first member of an extended Higgs sector, which would be a portal into New Physics. The clarification of this question through the search for additional Higgs bosons is of highest importance.

Extended Higgs sectors are naturally predicted by supersymmetry (SUSY). The structure of the Higgs sector in the two most basic variants, the Minimal (MSSM) and Next-to-Minimal Supersymmetric Standard Model (NMSSM) is illustrated in Table 1. The MSSM features three neutral Higgs bosons, jointly denoted as $\phi$, the NMSSM contains five such bosons. In any viable model, the observed state at $125 \mathrm{GeV}$ must be identified with one of the bosons in the Higgs sector, and its properties must resemble closely those of the SM Higgs boson. In case of the MSSM, it is generally most promising to identify the lightest Higgs boson, $h$, with the measured state, and the additional bosons would thus be expected at higher masses. In the NMSSM case, several associations are possible, so that additional Higgs bosons both heavier and lighter than $125 \mathrm{GeV}$ are feasible.

Table 1: Structure of the Higgs sector in the MSSM and NMSSM models.

\begin{tabular}{rcccc}
\hline Model & Structure & CP-even & CP-odd & Charged \\
\hline MSSM & 2 doublets & $\mathrm{h}, \mathrm{H}$ & $\mathrm{A}$ & $\mathrm{H}^{ \pm}$ \\
NMSSM & 2 doublets + 1 singlet & $\mathrm{h}_{1}, \mathrm{~h}_{2}, \mathrm{~h}_{3}$ & $\mathrm{a}_{1}, \mathrm{a}_{2}$ & $\mathrm{~h}^{ \pm}$ \\
\hline
\end{tabular}

\section{Search for MSSM Higgs bosons}

With the lightest MSSM Higgs boson h placed at a mass of $125 \mathrm{GeV}$ with SM-like properties, the other bosons $\mathrm{H}$ and A should be degenerate at higher masses. For values of the MSSM parameter $\tan \beta$ significantly above unity, their by far dominant decay channel would be $\mathrm{H} / \mathrm{A} \rightarrow \mathrm{b} \overline{\mathrm{b}}$. Searching for such a signal is very challenging due to the huge background from QCD multijet production. It is most promising to search for Higgs production in association with one or more additional $\mathrm{b}$ quarks, because this cross section would be enhanced by a factor of $2 \tan ^{2} \beta$. This selection also reduces the QCD multijet background. The signal-to-background ratio is thus improved. Example Feynman diagrams are shown in Figure 1. A dedicated trigger is essential to reduce the rate at the online level. The recent CMS analysis [4] is based on a trigger requiring at least two jets of high transverse momentum $\left(p_{\mathrm{T}}\right)$, and at least two tight online $\mathrm{b}$ tags in the event.

The analysis requires the three leading jets to pass $p_{\mathrm{T}}$ thresholds of 80,70 and $20 \mathrm{GeV}$, respectively, and to satisfy strong $b$ tagging requirements. The data sample thus selected contains about $69 \mathrm{k}$ events. A signal would show as a peak in the distribution of the invariant mass of the two leading jets $\left(M_{12}\right)$, as shown in Figure 2 (left) for three different Higgs masses. In addition, a condensed event $b$ tag estimator is computed from the secondary vertex mass sums of the three leading jets, which can take discrete values from $0-8$ and is used as second variable. The dominant 
background contribution from QCD multijet production is estimated from control data in which only two of the three leading jets are $b$ tagged.
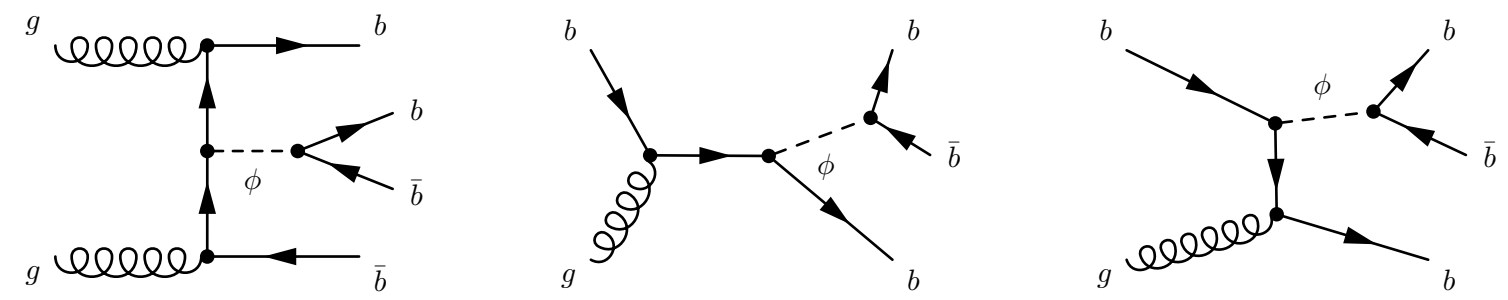

Figure 1: Example Feynman diagrams of the signal processes.
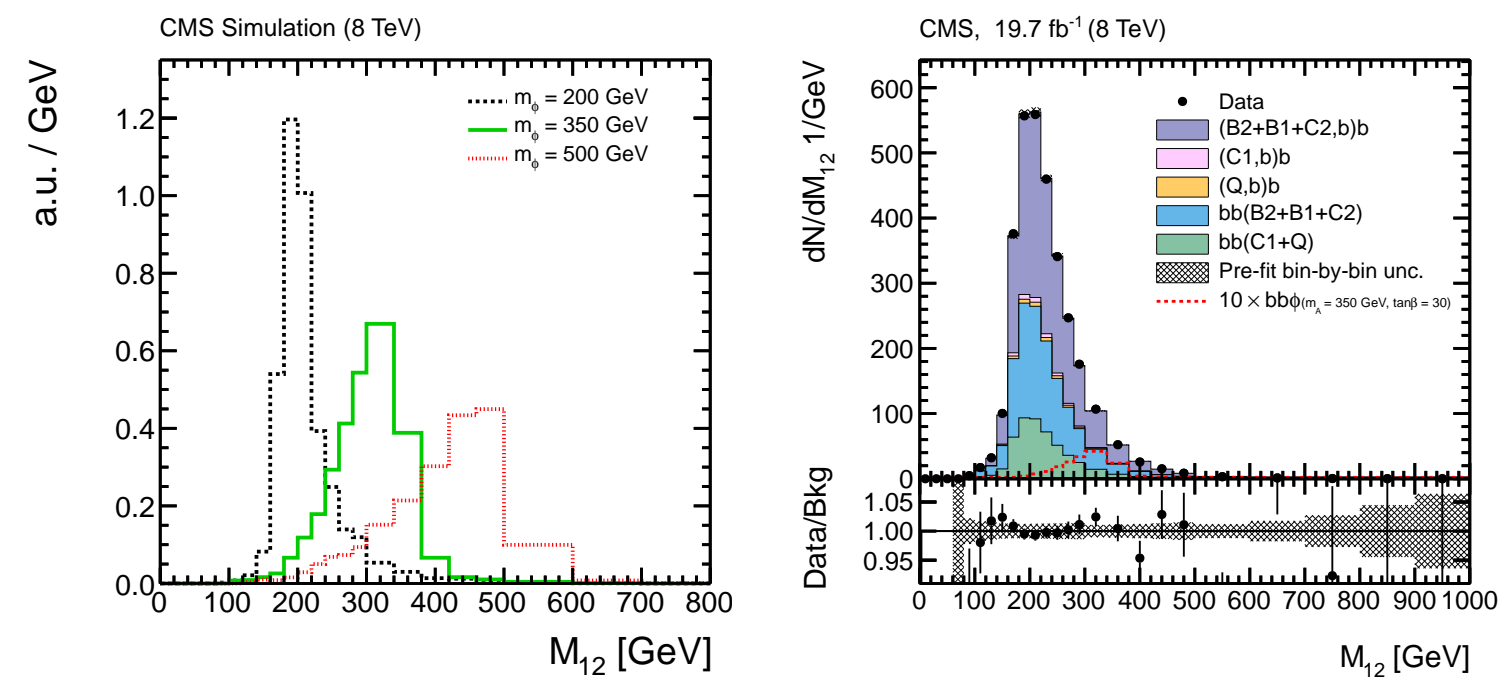

Figure 2: Left: The $M_{12}$ projection of the simulated signal templates for Higgs boson masses of $m_{\phi}=$ 200, 350 and $500 \mathrm{GeV}$. Right: Projection of the dijet mass $M_{12}$ in the data, together with the corresponding projections of the fitted background templates. The hatched area shows the total bin-by-bin background uncertainty of the templates prior to the fit. For illustration, the signal contribution expected in the $m_{\mathrm{h}}^{\max }$ benchmark scenario of the MSSM with $m_{\mathrm{A}}=350 \mathrm{GeV}, \tan \beta=30$, and $\mu=+200 \mathrm{GeV}$ is overlayed, scaled by a factor 10 for better readability. In addition, the ratio of data to the background estimate is shown at the bottom.

The measured invariant mass spectrum is shown in Figure 2 (right). The data are well described by the background model, which is composed of five templates representing different flavor combinations that are derived from the control sample with two $b$ tags. There is no indication of a signal. Upper limits on the cross section times branching fraction are shown in Figure 3 (left). They are translated into exclusion limits in the MSSM parameters $\tan \beta$ and $m_{\mathrm{A}}$ in the context of the $m_{\mathrm{h}}^{\bmod +}$ benchmark scenario [6], which are shown in Figure 3 (right). In this case the results from the previous CMS analysis at $7 \mathrm{TeV}$ [5] are included as well. The upper limits on $\tan \beta$ range from about 14-50. They are the most stringent limits obtained in this channel to date.

\section{Search for light NMSSM Higgs bosons}

The existence of light Higgs bosons with mass $m_{\mathrm{H}}<100 \mathrm{GeV}$ and standard couplings has 
CMS, $19.7 \mathrm{fb}^{-1}(8 \mathrm{TeV})$

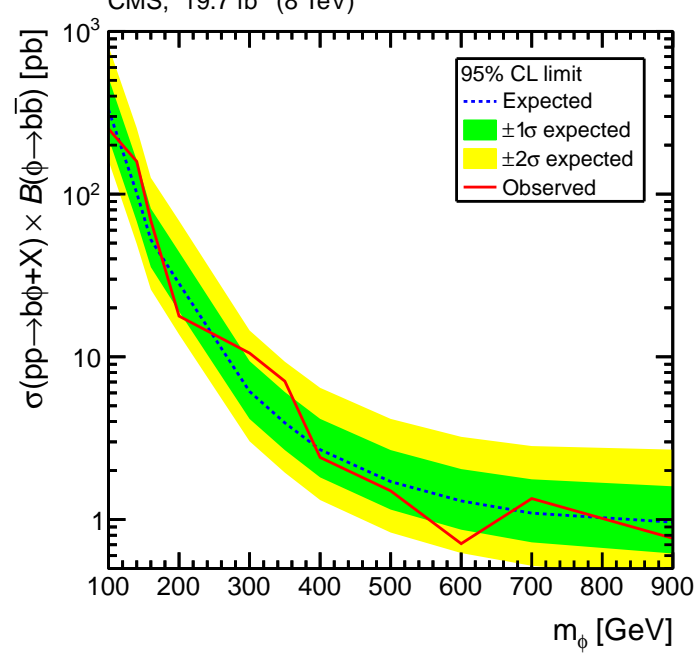

CMS, $19.7 \mathrm{fb}^{-1}(8 \mathrm{TeV})+4.9 \mathrm{fb}^{-1}(7 \mathrm{TeV})$

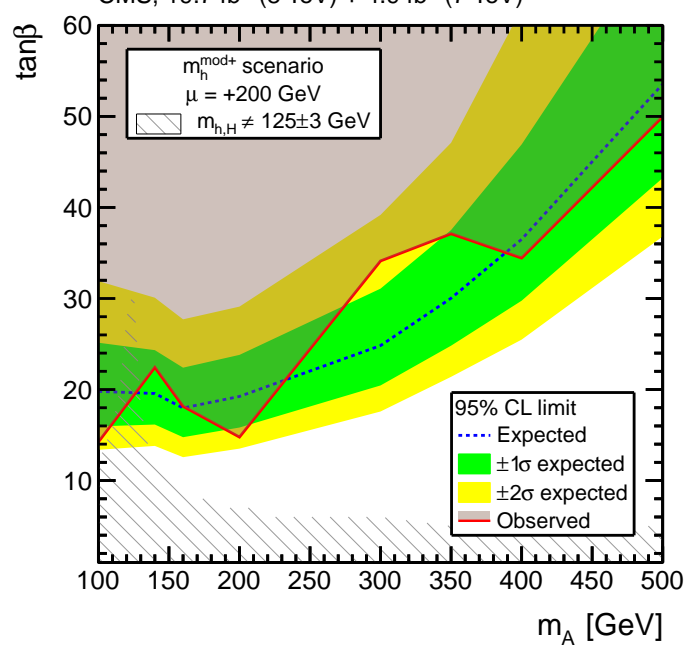

Figure 3: Left: Expected and observed upper limits at 95\% confidence level (CL) on $\sigma(p p \rightarrow \phi+\mathrm{X}) \times$ $\mathscr{B}(\phi \rightarrow b \bar{b})$ as a function of $m_{\phi}$, where $\phi$ denotes a generic neutral Higgs-like state. Right: Expected and observed upper limits at 95\% CL for the MSSM parameter $\tan \beta$ versus $m_{\mathrm{A}}$ in the $m_{\mathrm{h}}^{\bmod +}$ benchmark scenario with $\mu=+200 \mathrm{GeV}$ [6]. The excluded parameter space (observed limit) is indicated by the shaded area. Regions where the mass of neither of the CP-even MSSM Higgs bosons h or H is compatible with the discovered Higgs boson of $125 \mathrm{GeV}$ within a range of $3 \mathrm{GeV}$ are marked by the hatched areas.

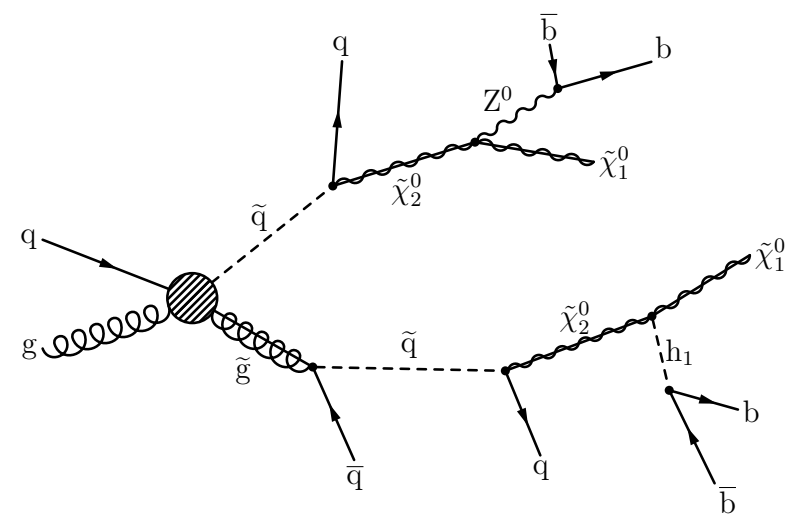

Figure 4: Feynman diagram of squark-gluino production with subsequent cascade decays via neutralinos into $h_{1}$.

already been largely excluded at LEP [7]. Nevertheless, within the NMSSM, scenarios are possible in which these constraints are evaded. In the "modified P4 benchmark scenario" [8, 9], the observed state at $125 \mathrm{GeV}$ is identified with the $\mathrm{h}_{2}$ which has SM-like properties. The significantly lighter $\mathrm{h}_{1}$ has a large singlet component resulting in strongly reduced couplings to SM vector bosons and fermions, and thus could have escaped detection at LEP. On the other hand, it would be copiously produced in supersymmetric cascades, as depicted in Figure 4, and it would dominantly decay to $\mathrm{b} \overline{\mathrm{b}}$. 


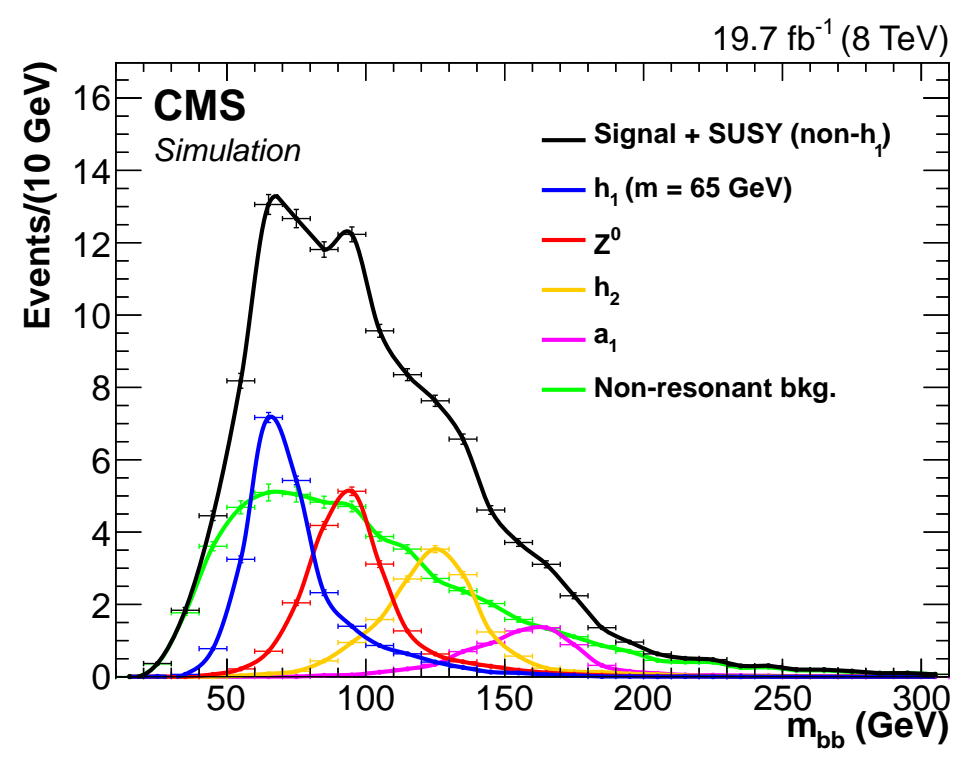

Figure 5: Signal + non- $h_{1}$ SUSY prediction of the modified P4 scenario with $m_{h_{1}}=65 \mathrm{GeV}$ and normalized to the integrated luminosity of $19.7 \mathrm{fb}^{-1}$.

This possibility has been for the first time explored at the LHC by the CMS experiment [10]. The analysis requires large hadronic activity of $H_{\mathrm{T}}>750 \mathrm{GeV}$, where $H_{\mathrm{T}}$ is defined as the scalar $p_{\mathrm{T}}$ sum of all jets with $p_{\mathrm{T}}>40 \mathrm{GeV}$ and $|\eta|<3$. Furthermore, large missing energy $E_{\mathrm{T}}^{\text {miss }}>200 \mathrm{GeV}$, two energetic jets as expected from squark decays with $p_{\mathrm{T}}>250$ and $100 \mathrm{GeV}$, respectively, and two additional b-tagged jets with $p_{\mathrm{T}}>25 \mathrm{GeV}$ are required. The expected signal model from the SUSY cascade is shown in Figure 6; besides a peak from the $h_{1}$, also signals from the $h_{2}, a_{1}$ and $Z$ bosons should appear, as well as a non-resonant contribution. The background from SM processes is dominated by $\mathrm{t} \overline{\mathrm{t}}+$ jets and QCD multijet production.

The measured distribution of the invariant mass of the two b-jet candidates is shown in Figure 6 (left). The expected $\mathrm{h}_{1}$ signal according to the modified $\mathrm{P} 4$ scenario is shown as a red curve, while the other contributions from the SUSY cascade are displayed as a blue dotted line. The data do not exhibit any such structure; they are well described by the SM background expectation.

By inclusion of the expected signal contribution in the fit, upper limits on cross section times branching fraction are derived at $95 \% \mathrm{CL}$ in the $\mathrm{h}_{1}$ mass range of $30-100 \mathrm{GeV}$, which are shown in Figure 6 (right). The prediction of the modified P4 scenario is also shown for squark and gluino masses of $1 \mathrm{TeV}$; this model is excluded by the data at 95\% CL over the whole mass range. This measurement complements an earlier CMS search for production of the $\mathrm{H}(125)$ boson in SUSY cascades [11].

\section{Summary}

Searches for Higgs bosons beyond the SM have been performed in the $b \bar{b}$ decay channel. These studies are unique at the LHC. No signal has been found for such a boson produced in combination with at least one more $b$ jet in the mass range of 100-900 GeV. Upper limits on the MSSM parameter $\tan \beta$ ranging from 14-50 have been derived up to a mass $m_{\mathrm{A}}$ of $500 \mathrm{GeV}$. A 

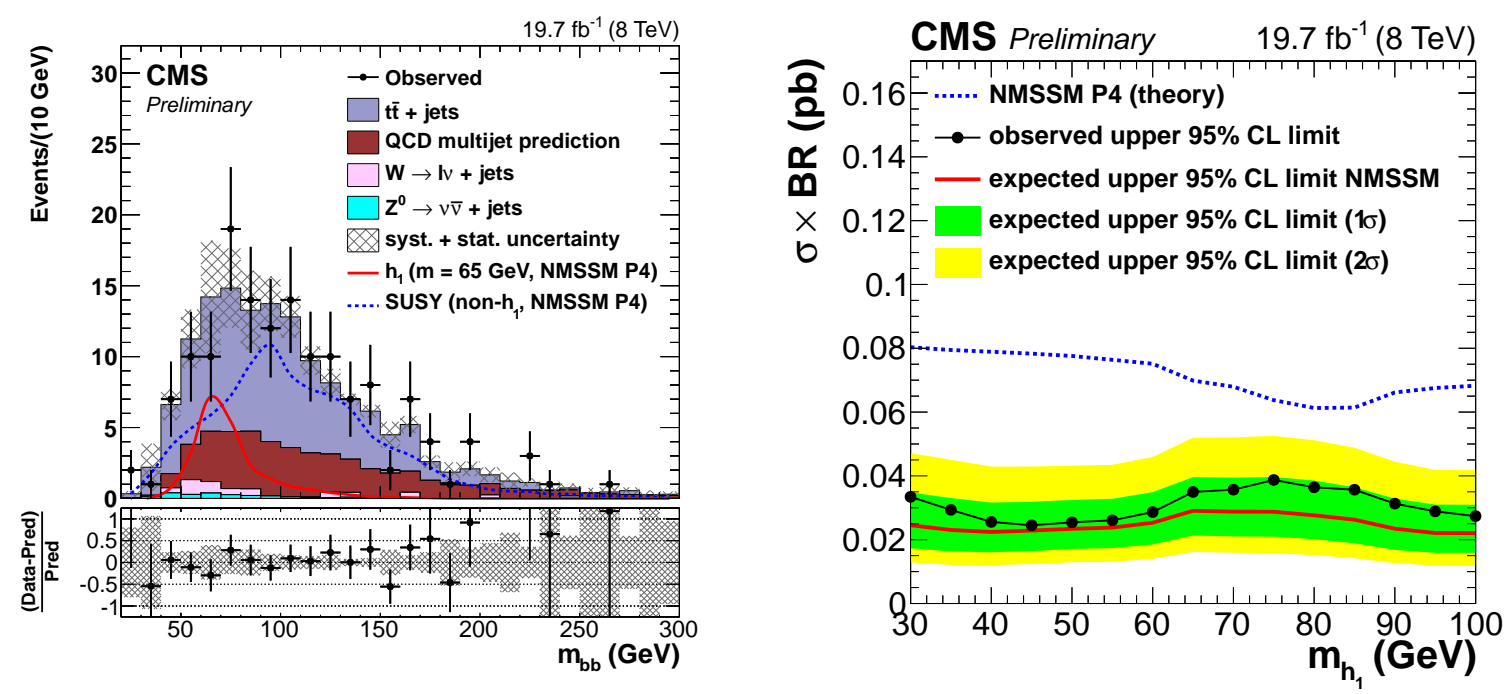

Figure 6: Left: Background-only fit result of the invariant mass distribution of the selected b-tagged jets, where the SM background contributions are stacked. The theoretical $h_{1}$ and non- $h_{1}$ contributions are overlaid to illustrate the sensitivity of the analysis to the modified P4 scenario expectation. Right: Upper limits for the NMSSM cross section times branching fraction into $b \bar{b}$ in comparison to the theoretical expectations from the NMSSM modified P4 scenario (blue dashed line).

light NMSSM Higgs boson produced in supersymmetric cascades has been searched for in the mass range of $30-100 \mathrm{GeV}$. No signal is found. The NMSSM modified P4 scenario with squark and gluino masses of $1 \mathrm{TeV}$ is excluded at $95 \% \mathrm{CL}$ in this whole mass range.

\section{References}

[1] ATLAS Collaboration, "Observation of a new particle in the search for the Standard Model Higgs boson with the ATLAS detector at the LHC", Phys. Lett. B 716 (2012) 1.

[2] CMS Collaboration, "Observation of a new boson at a mass of $125 \mathrm{GeV}$ with the CMS experiment at the LHC”, Phys. Lett. B 716 (2012) 30.

[3] CMS Collaboration, "Observation of a new boson with mass near $125 \mathrm{GeV}$ in pp collisions at $\sqrt{s}=7$ and 8 TeV”, JHEP 06 (2013) 081.

[4] CMS Collaboration, "Search for neutral MSSM Higgs bosons decaying into a pair of bottom quarks", (2015). arXiv:1506.08329. Submitted to JHEP.

[5] CMS Collaboration, "Search for a Higgs boson decaying into a b-quark pair and produced in association with b quarks in proton-proton collisions at 7 TeV”, Phys. Lett. B 722 (2013) 207.

[6] M. Carena et al., "MSSM Higgs boson searches at the LHC: benchmark scenarios after the discovery of a Higgs-like particle”, Eur. Phys. J. C 73 (2013) 2552.

[7] ALEPH, DELPHI, L3 and OPAL Collaborations, LEP Working Group for Higgs Boson Searches, "Search for neutral MSSM Higgs bosons at LEP”, Eur. Phys. J. C 47 (2006) 547.

[8] A. Djouadi et al., "Benchmark scenarios for the NMSSM", JHEP 07 (2008) 002.

[9] O. Stål and G. Weiglein, "Light NMSSM Higgs bosons in SUSY cascade decays at the LHC", JHEP 01 (2012) 071. 
[10] CMS Collaboration, "Search for a light NMSSM Higgs boson produced in supersymmetric cascades and decaying into a b-quark pair", CMS PAS HIG-14-030, http://cds.cern.ch/record/2002557.

[11] CMS Collaboration, "Searches for supersymmetry using the MT2 variable in hadronic events produced in pp collisions at $8 \mathrm{TeV}$ ”, JHEP 05 (2015) 078. 